East African Medical Journal Vol. 86 No. 1 January 2009

BURDEN AND PATTERN OF CANCER IN WESTERN KENYA

C.N. Tenge MBChB, MMed (Paed), Lecturer, Department of Child Health and Paediatrics, R. T. Kuremu, MBChB, MMed (Surg), MMed Sc. (Paed Surg), FCS (ECSA) Senior Lecturer, Department of Surgery and Anaesthesiology, Moi University, School of Medicine, N.G. Buziba, MBChB, MMed (Pathol), Senior Lecturer, Department of Haematology and Blood Transfusion, K. Patel, BSc. (Microbiol), MSc. (Applied Biology), DCA (Clinical Analysis) Assistant Lecturer, Department of Immunology, Moi University, School of Medicine and P. A. Were, KRCHN, Higher Dip. (Palliative Care) Nursing Officer - Oncology Department, Moi Teaching and Referral Hospital, P.O. Box 4606 - 30100, Eldoret, Kenya

Request for reprints to: Dr. C. N. Tenge, Department of Child Health and Paediatrics, School of Medicine, Moi University, P.O. Box 4606 - 30100, Eldoret, Kenya

\title{
BURDEN AND PATTERN OF CANCER IN WESTERN KENYA
}

\author{
C.N. TENGE, R.T. KUREMU, N.G. BUZIBA, K. PATEL and P. A. WERE
}

\begin{abstract}
Background: Cancer regisries worldwide have evolved to provide useful information on the burden and diversity of the patterns of cancer, information that is vital for establishing appropriate programmes for disease management. Population based data on cancer in western Kenya as captured in the Eldoret cancer registry established in 1999 is analysed and reported in this paper.

Objective: To determine the burden and pattern of cancer in Western Kenya by use of data from the Eldoret cancer registry.

Design: Retrospective study.

Setting: The cancer registry located in the Department of Haematology at the Moi University, School of Medicine situated at the Moi Teaching and Referral Hospital, Eldoret, Kenya. The hospital has a catchment population of 13 to 15 million people forming about $40 \%$ of the Kenyan population.

Results: A total of 5,366 patients were diagnosed to have cancer and attended to at the MTRH and other hospitals in Eldoret during the period between January 1999 and December 2006 giving an average of 671 cases per year. Among those treated 2,699 were males and 2,667 were females giving a M: F ratio of 1:1. About $21 \%$ of the patients had haematological malignancies with non-Hodgkins lymphoma being the most common. Another 79\% of the patients had solid tumours with cancer of the oesophagus being the commonest. Cancer of the cervix and prostrate were the commonest among the females and males respectively. A general increase in the number of patients with Kaposis sarcoma associated with HIV/AIDS pandemic was observed.

Conclusion: The burden of cancer is a significant health problem in western Kenya and there is need for the development of a comprehensive cancer care programme in the region to address the growing problem.
\end{abstract}

\section{INTRODUCTION}

Cancer is a worldwide disease and one of the leading causes of morbidity and mortality in the developed world where the burden of disease is accurately determined. In the developing countries, determination of the burden and pattern has not reached the same level as is the case in the west. Projections indicate an expected yearly incidence of 15 to 20 million new cases worldwide with a staggering 50 to $60 \%$ occurring in the developing world (1-4). To handle this expected epidemic, adequate information on burden and pattern of cancer needs to be available in order to promote effective decision making.

Knowledge on cancersin Africa is unfortunately very scanty (5). Cancer as a disease entity has not been given the attention it deserves hence the limited knowledge. Until recently, available information 
was based on hospital reviews by clinicians and pathologists (6). The need to embrace and manage population based registries is therefore self evident. Information generated from such registries will be important in planning and setting up programmes in cancer management.

The Eldoret cancer registry was established in 1999 and uses the active case finding approach from hospital records, pathology reports and death certificates in MTRH and other hospitals within the Uasin Gishu district to identify and register cancer cases. A significant proportion of patients are referrals from hospitals in the greater western Kenya region. Secondary data reviewed from this registry therefore provides information on the burden and pattern of cancer in western Kenya. It is not clear what proportion is captured but it gives an estimated burden in the catchment region which hosts about $40 \%$ of the Kenyan population.

\section{MATERIALS AND METHODS}

Secondary data from the Eldoret Cancer Registry located in the Department of Haematology at the Moi University School of Medicine located at the Moi Teaching and Referral Hospital, Eldoret were reviewed.

A pathologist is in charge of the registry which has a registrar and secretary as full time staff and engages Health Information and Records officers who collect theinformation from the hospital records department, pathology department, in-patient wards, the Eldoret Hospice and also from death certificates. Cases are identified from the disease index (completed by records staff in ICD-10) and details of cases abstracted from case records. Cases are coded in the registry and data entry and management done by the CANREG-3 system, a software developed by IARC and designed for population based cancer registries.

The frequency and pattern of distribution of the cases of cancer was determined and classification done. The types of cancer were broadly classified into haematological and nonhaematological (solid tumours) malignancies. The haematological malignancies include Hodgkins and non-Hodgkins lymphoma, acute as well as chronic leukemia and others like multiple myeloma, plasmacytoma, myelopdysplastic syndrome and essential thrombocytosis. The solid tumours or non-haematological malignancies include cancers of various organs like the brain, thyroid, oesophagus, stomach, kidneys, cervix, skin, bone and muscle.

\section{RESULTS}

A total of 5,366 patients were diagnosed to have cancer and attended to at the MTRH and other hospitals in Uasin Gishu district during the period between January 1999 to December 2006. 2,699 patients were males and 2,667 were females giving a M:F ratio of 1: 1 . Figure 1 shows the yearly distribution of the number of patients diagnosed with cancer giving an average of 671 cases per year.

There were $1,108(20.6 \%)$ patients with haematological malignancies. Figure 2 shows the different types of haematological malignancies and the gender distribution. Lymphomas were the most common $(11.9 \%)$, followed by acute leukaemia $(4.0 \%)$ and chronic leukaemia $(3.2 \%)$. There were $4,258(79.4 \%)$ patients with non-haematological malignancies or solid tumours. Figure 3 shows the different types of non-haematological malignancies and the gender distribution.

Figure 1

Annual distribution of the number of patients diagnosed with cancer

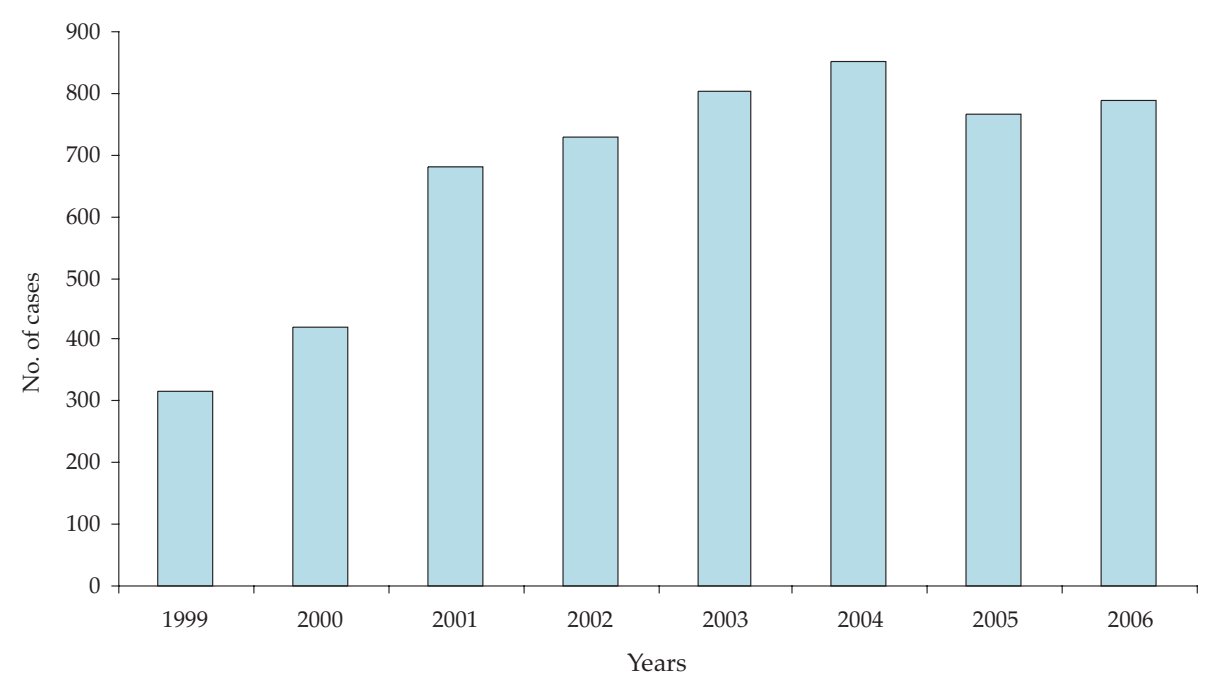


Figure 2

Haematological malignancies

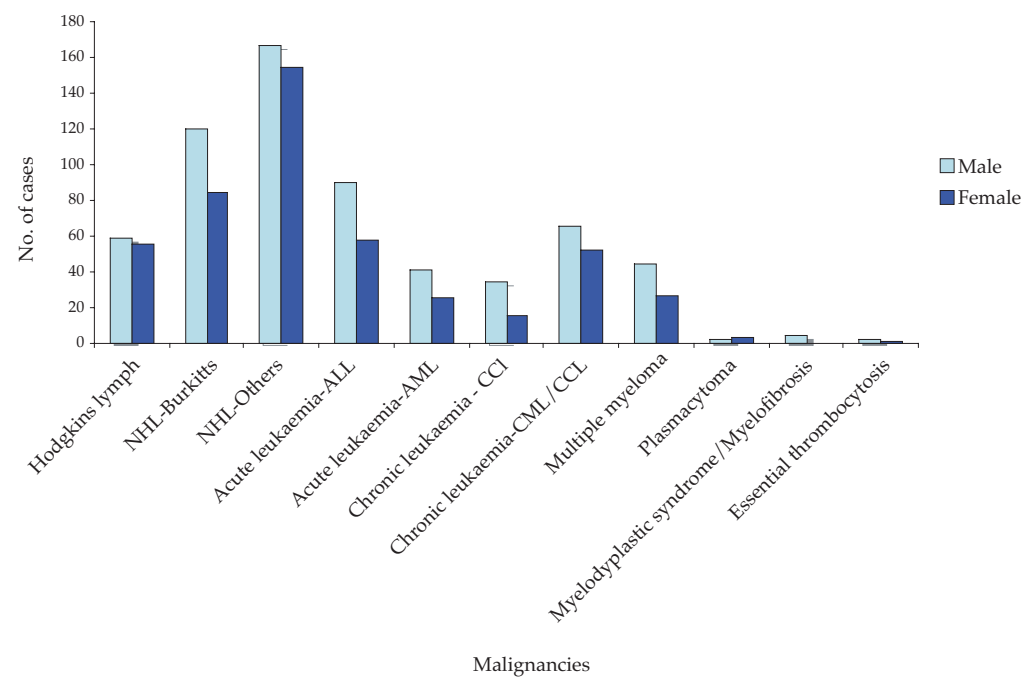

Figure 3

Non-haematological malignancies

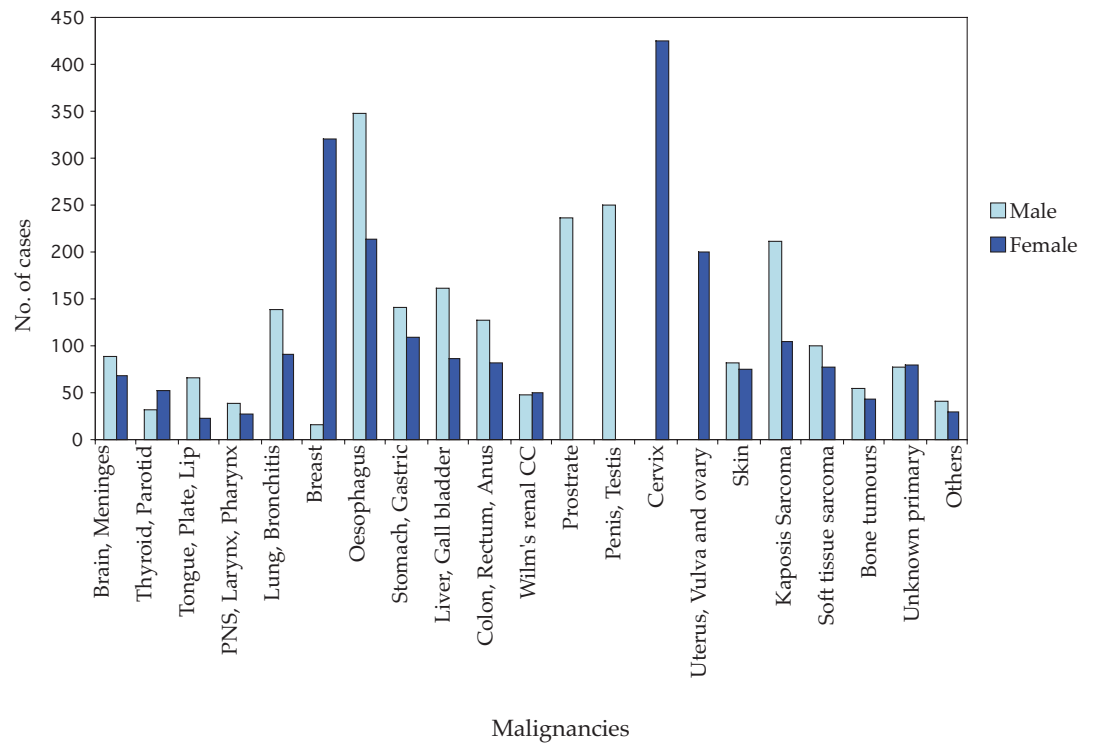

In all the patients with non-haematological malignancies, cancer of the oesophagus was the most common $(10.5 \%)$, followed by cancer of the breast $(6.2 \%)$ and Kaposis sarcoma (5.9\%). Cancer of the cervix $(10.0 \%)$ and prostate $(5.5 \%)$ were common among the females and males respectively.

\section{DISCUSSION}

Cancer management is one of the health needs which do not receive adequate attention from the health care providers and policy makers in the developing countries $(2,4)$. Most of the health care providers areill prepared toeffectively recognise and take appropriate measures when faced with cancer patients. This results from a combination of inadequate knowledge, poor attitude, lack of appropriate skills and facilities to manage the patients effectively $(2,4)$.The appalling poverty, poor hygiene and complex social dynamics contribute to the issue of affordability and accessibility and sincethe patients have to bear the cost of treatment, most of them initially opt to utilise alternative and traditional systems of medicine and when these have failed, they eventually end up in the appropriate health institutions with late stage incurable disease whereby the only option is palliative care $(3,4)$.

The findings of the study show an increase in the number of patients attended to at MTRH and other hospitals in Uasin Gishu district from 1999 to 2004 and then a slight decline in 2005 and 2006 (Figure 1). The possible reasons for the increase in the number of cases include the establishment of a functional oncology service which encouraged the referral of patients for care and the existence of a cancer registry 
that improved record keeping. The decline could be due to the establishment of an outreach oncology service based at five distant sites in a radius of about $150 \mathrm{~km}$ from Eldoret in 2005. A significant number of patients are seen at the satellite sites without ever coming to MTRH. The oncology services at these sites are integrated with the comprehensive care programme run by the Academic Model for the Prevention and Treatment of HIV / AIDS (AMPATH). The apparent success of the outreach services has opened up a significant opportunity of realising the desire to effectively serve the rural poor who are not able to access centralized services far away from them.

Among the haematological malignancies, the lymphomas were the most common $(11.9 \%)$ followed by the acute leukemia (4.0\%). Similar to occurrences elsewhere, these malignancies are commonly seen in the young population. The chronic leukemia (3.2\%) is seen in the older population.

Among the solid tumours cancer of the oesophagus $(10.5 \%)$, cervix $(7.9 \%)$, breast $(6.2 \%)$, Kaposis sarcoma $(5.9 \%)$ and prostrate $(4.4 \%)$ were the most common. These results are similar to findings of studies done elsewhere in Africa. The good news for this region is that most of these cancers are curable if detected early and treated appropriately $(4,6)$. Majority of these cancers are also preventable hence correct application of available information could prevent up to a third of the cases $(1,2,5,7,8)$. A well organised oncology service can therefore make a major positive change.

There is a significant increase in the number of patients accessing care both within the MTRH and at the satellite clinics yet the oncology services still face serious challenges. This is a resource constraint region with few health care providers to serve the large population, chemotherapeutic drugs are expensive and radiotherapy is yet to be established. Negative believes and attitudes still play a significant role in influencing the accessibility of health services. These are among the challenges to contend with and like most of the other resource constrained regions it looks forward to benefiting from the global initiatives to fight cancer $(3,9)$.

Development of a comprehensive oncology/ cancer care programme is necessary to promote effective management of patients with cancer in western Kenya. The first role of the programme should be to sensitise the relevant authorities on the position of the oncology services in raising the well being of the population. There is also a need for inclusion of information on cancer management in training curricular for health care professionals. The programme should be able to support the management of patients through strengthening the already existing national hospital insurance fund (NHIF), donations and research activities. Other necessary measures include proper record keeping through creation of an oncology database, enhancing screening activities, development and use of treatment protocols suitable for resource limited settings, establishment of radiotherapy services and proper coordination between various disciplines involved in cancer care. As part of the efforts to work with others committed to fighting cancer, a collaboration between MTRH (Kenya), Moi University School of Medicine (Kenya), Indiana University (USA), Brown University (USA) and Oregon university (USA) has been set up to put in motion the above recommendations. More local stakeholders need to come on board to boost these efforts.

\section{ACKNOWLEDGEMENTS}

To the management of MTRH, AMPATH, and Moi University - School of Medicine, Eldoret hospice and Dr. M. Karim of Eldochem chemist for the support they give to the oncology team as it provides care to cancer patients. We are grateful to our collaborators Prof. J. Mamlin, Prof. A. Rosmarin, Prof. P. Loehrer, Prof.H. Cardenes, Prof. R. Einterz, Dr. M.Strother and Dr. E. Chirchella for their guidance and willingness to assist in the development of a comprehensive cancer care programme. Thanks to R. Chemokos, C. Nyongesa and M. Ondwassy for their services and E. Korir for data analysis.

\section{REFERENCES}

1. Matters, C.D. and Loncar, D. Projections of global mortality and burden of diseases from 2002 to 2030 . Plos. Med. 2006; 3:e 442.

2. P. Kanavos. The rising burden of cancer in the developing world. Annals of Oncol. 2006; 17 (supl 8): viii 15 - viii 23.

3. Magrath, I. and Litrak, J. Cancer in developing countries: Opportunities and challenges. J. Nat. Cancer Inst. 1993; 85: 862-874.

4. Pal, S.K. and Mittal, B. Fight against cancer in countries with limited resources:- the post genomic era scenario. Asian Pac. J. Cancer Prev. 2004; 5: 328-333.

5. Ngoma, T. World Health Organization. Cancer priorities in developing countries. Ann Oncol. 2006; 17 (Suppl 8): viii 9 - viii 14.

6. Cancer in Africa; Epidemiology and Prevention, IARC Scientific Publication No. 53. Edited by D.M. Parkin et al. IARC Press Lyon, France. 2003.

7. MellisdedtH.Cancerinitiativesindeveloping countries. Ann. Oncol. 2006; 17(Suppl 8): viii 24-viii 31.

8. Jones, L.A. et al. Between and within: - International perspectives of cancer and health disparities. J. Clin. Oncol. 2006; 24: 2204-2208.

9. Stayce, B. and Chrisitne, T. Cancer in the developing world. How should care be derived and research conducted? J. National Cancer Institute. 2003; 95: 1651-1653. 